\title{
Cardiac involvement in Beagle-based canine X-linked muscular dystrophy in Japan (CXMD): electrocardiographic, echocardiographic, and morphologic studies
}

\author{
Naoko Yugeta ${ }^{\dagger 1,2}$, Nobuyuki Urasawa ${ }^{\dagger 2}$, Yoko Fujii ${ }^{1}$, Madoka Yoshimura ${ }^{2}$, \\ Katsutoshi Yuasa2, Michiko R Wada², Masao Nakura3, Yoshiki Shimatsu², \\ Masayuki Tomohiro ${ }^{2}$, Akio Takahashi ${ }^{4}$, Noboru Machida ${ }^{5}$, Yoshito Wakao ${ }^{1}$, \\ Akinori Nakamura ${ }^{2}$ and Shin'ichi Takeda*2
}

Address: ${ }^{1}$ Department of Surgery I, School of Veterinary Medicine, Azabu University, Fuchinobe, Sagamihara, Kanagawa, 229-8501, Japan, ${ }^{2}$ Department of Molecular Therapy, National Institute of Neuroscience, National Center of Neurology and Psychiatry, 4-1-1 Ogawa-higashi, Kodaira, Tokyo 187-8502, Japan, ${ }^{3}$ Chugai Research Institute for Medical Science, Inc., 6598 Toyoda, Suwa, Nagano 392-0016, Japan, ${ }^{4}$ Division of Laboratory Animal Resources, National Institute of Neuroscience, National Center of Neurology and Psychiatry, 4-1-1 Ogawa-higashi, Kodaira, Tokyo 187-8502, Japan and 5epartment of Veterinary Pathology, Faculty of Agriculture, Tokyo University of Agriculture and Technology, 3-5-8 Saiwai-cho, Fuchu, Tokyo 183-8509, Japan

Email: Naoko Yugeta - yugeta@b-star.jp; Nobuyuki Urasawa - urasawan@amber.plala.or.jp; Yoko Fujii - fujii@dc4.so-net.ne.jp; Madoka Yoshimura - yoshimur@ncnp.go.jp; Katsutoshi Yuasa - yuasa@ncnp.go.jp; Michiko R Wada - wadarin@ncnp.go.jp; Masao Nakura - nakuramso@chugai-pharm.co.jp; Yoshiki Shimatsu - shimatsu@ncnp.go.jp; Masayuki Tomohiro - tomhromy@banyu.co.jp; Akio Takahashi - akio_tk@ncnp.go.jp; Noboru Machida - machida@cc.tuat.ac.jp; Yoshito Wakao - wakao@azabu-u.ac.jp; Akinori Nakamura - anakamu@ncnp.go.jp; Shin'ichi Takeda* - takeda@ncnp.go.jp

* Corresponding author †Equal contributors

\section{Published: 04 December 2006}

BMC Cardiovascular Disorders 2006, 6:47 doi:10.1 186/147|-226I-6-47

This article is available from: http://www.biomedcentral.com/I47|-226I/6/47

(C) 2006 Yugeta et al; licensee BioMed Central Ltd.

This is an Open Access article distributed under the terms of the Creative Commons Attribution License (http://creativecommons.org/licenses/by/2.0), which permits unrestricted use, distribution, and reproduction in any medium, provided the original work is properly cited.
Received: I 4 July 2006

Accepted: 04 December 2006

\begin{abstract}
Background: Cardiac mortality in Duchenne muscular dystrophy (DMD) has recently become important, because risk of respiratory failure has been reduced due to widespread use of the respirator. The cardiac involvement is characterized by distinctive electrocardiographic abnormalities or dilated cardiomyopathy, but the pathogenesis has remained obscure. In research on DMD, Golden retriever-based muscular dystrophy (GRMD) has attracted much attention as an animal model because it resembles DMD, but GRMD is very difficult to maintain because of their severe phenotypes. We therefore established a line of dogs with Beagle-based canine $X$-linked muscular dystrophy in Japan (CXMD, and examined the cardiac involvement.

Methods: The cardiac phenotypes of eight CXMD, and four normal male dogs 2 to 21 months of age were evaluated using electrocardiography, echocardiography, and histopathological examinations.

Results: Increases in the heart rate and decreases in $\mathrm{PQ}$ interval compared to a normal littermate were detected in two littermate CXMD, dogs at 15 months of age or older. Distinct deep $Q$-waves and increase in $Q / R$ ratios in leads II, III, and aVF were detected by 6-7 months of age in all CXMD, dogs. In the echocardiogram, one of eight of CXMD, dogs showed a hyperechoic lesion in the left ventricular posterior wall at 5 months of age, but the rest had not by 6-7 months of age. The left ventricular function in the echocardiogram indicated no abnormality in all CXMD, dogs by 6-7 months of age. Histopathology revealed myocardial fibrosis, especially in the left ventricular posterobasal wall, in three of eight CXMD, dogs by 21 months of age.
\end{abstract}




\begin{abstract}
Conclusion: Cardiac involvement in CXMD, dogs is milder and has slower progression than that described in GRMD dogs. The distinct deep Q-waves have been ascribed to myocardial fibrosis in the posterobasal region of the left ventricle, but our data showed that they precede the lesion on echocardiogram and histopathology. These findings imply that studies of CXMD, may reveal not only another causative mechanism of the deep Q-waves but also more information on the pathogenesis in the dystrophin-deficient heart.
\end{abstract}

\section{Background}

Duchenne muscular dystrophy (DMD) is a common and lethal genetic disease characterized by progressive muscle wasting. It is an X-linked recessive disorder caused by mutations in the dystrophin gene, which encodes a cytoskeletal protein, dystrophin [1]. The absence of dystrophin is accompanied by a loss of dystrophin-glycoprotein complex at the sarcolemma and results in progressive degeneration of skeletal and cardiac muscle with fibrotic tissue replacement and fatty infiltration $[2,3]$. The onset of the disease occurs between 2 and 5 years of age, and most patients die of respiratory or cardiac failure [4,5]. Cardiac involvement, which occurs commonly in DMD patients, has increasingly become an important cause of death because recent clinical progress has reduced the risk of death due to respiratory failure [6,7].

Like dystrophin-deficient skeletal muscle, dystrophindeficient cardiac muscle is replaced by fibrotic or fatty tissue, especially in the left ventricular posterobasal wall region [8-11]. Atrophic changes with loss of striation, vacuolation, fragmentation, or nuclear degeneration in the myocardium have also been reported [12]. Progressive involvement of the left ventricle leads to wall motion abnormality and results in dilated cardiomyopathy. In DMD patients, the electrocardiogram (ECG) may show tall R-waves in the right precordial leads, deep Q-waves in leads I, aVL, V5-6 or II, III, and aVF [8-13], as well as an increased heart rate, shortened PQ (PR) interval, conduction abnormalities or arrhythmias such as sinus arrhythmia, atrial ectopic beats, and ventricular premature complexes in DMD patients [13-16]. One of the electrocardiographic abnormalities, deep Q-waves, has been considered to be attributable to myocardial fibrosis $[8,9,17]$. Echocardiography indicates myocardial thickening, wall motion abnormalities, enlargement of the left ventricle, and left ventricular systolic and diastolic dysfunction. Hypokinesis of the posterobasal wall is consistent with the spreading fibrosis and significant decrease in the internal dimensions of the ventricles $[14,15]$. There are, however, many unresolved issues in cardiac involvement, such as the reason why the posterobasilar segment of the left ventricle is consistently the first lesion, whether extensive fibrosis involves the conduction system, the pathogenesis of inappropriate tachycardia or electrocardiographic abnormalities, and whether abnormal smooth muscle regulation affects the cardiomyopathy [18]. One way to clarify these problems is to study suitable animal models.

To date, the X-linked muscular dystrophy ( $m d x)$ mouse and the Golden retriever-based muscular dystrophy dog (GRMD) have been used for elucidation of the pathogenesis and development of therapy for DMD. The phenotypes of GRMD are more similar to DMD than that of the $m d x$ mouse [19-21], and GRMD also shows similar electrocardiographic findings and progressive cardiomyopathy comparable to the cardiac involvement of DMD patients [20-23]. In this respect, GRMD is a useful model to explore cardiac involvement, but GRMD is very difficult to maintain because of their severe phenotypes. Mild phenotypes can be expected in small sized dogs such as Beagle, indicated by the cross-bred study by Valentine et al. [20]. Moreover, medium-sized Beagle is easy to handle or raise than GRMD, therefore they have definite advantages in animal housing or welfare. Therefore, we established a Beagle-based dog colony named canine X-linked muscular dystrophy in Japan $\left(\mathrm{CXMD}_{\mathrm{J}}\right)$ [24]. In $\mathrm{CXMD}_{\mathrm{J}}$, involvement of the temporalis and limb muscles is observed from 2 months of age, and macroglossia, dysphagia, drooling, and joint contracture are apparent from 4 months of age; the phenotypes of $\mathrm{CXMD}_{\mathrm{J}}$ are thus almost comparable to GRMD [25]. In this study, we investigated the cardiac phenotypes in CXMD, using electrocardiography, echocardiography, and pathological examinations. Abnormalities on echocardiogram and cardiac pathology were detected from 12 months of age; however, the distinct deep Qwaves in leads II, III, and aVF on ECG were consistently observed by 6-7 months of age in all $\mathrm{CXMD}_{\mathrm{J}}$ dogs examined. The cardiac phenotypes of $\mathrm{CXMD}_{\mathrm{J}}$ were identical to but milder than those of GRMD described in the literature. Thus, CXMD may also be a suitable animal model for elucidation of the above-mentioned problems.

\section{Methods \\ Animals}

We imported frozen GRMD semen and artificially inseminated a Beagle bitch. The carriers produced were mated with unrelated Beagles, and a Beagle-based canine Xlinked muscular dystrophy $\left(\mathrm{CXMD}_{\mathrm{J}}\right)$ breeding colony was established [24]. In this study, four normal male and eight affected male dogs of the third generation (G3) between 2 to 21 months of age were examined. All of the affected and normal dogs were descendents of a single affected 
male, and were part of the $\mathrm{CXMD}_{\mathrm{J}}$ breeding colony at the General Animal Research Facility, National Institute of Neuroscience, National Center of Neurology and Psychiatry (NCNP) (Tokyo, Japan) or the Chugai Research Institute for Medical Science, Inc. (Nagano, Japan). The clinical and histopathological characteristics, except for cardiac involvement, of $\mathrm{CXMD}_{\text {I }}$ dogs were recently described [25]. These dogs were cared for and treated in accordance with the guidelines provided by the Ethics Committee for the Treatment of Laboratory Middle-Sized Animals of the National Institute of Neuroscience, NCNP (Tokyo, Japan) or the Ethics Committee for Treatment of Laboratory Animal of Chugai Pharmaceutical Co., Ltd. (Tokyo, Japan). These studies were also approved by the Ethics Committee for the Treatment of Laboratory Middle-Sized Animals of NCNP (approved No. 13-03, 14-03, 15-03, 16-03, 17-03, and 18-03). All experiments were performed with consideration for preventing unnecessary pain.

\section{Genotyping of CXMD, allele}

Each affected or normal male dog was identified by genotyping. A snapback method of single-strand conformation polymorphism analysis was used to determine the GRMD allele as described previously [26].

\section{Measurement of serum creatine kinase (CK)}

Blood samples were obtained from the cephalic vein at sacrifice. Serum CK level was measured by colorimetric assay using a FDC3500 clinical biochemistry analyzer (FujiFilm Medical Co., Tokyo, Japan).

\section{Electrocardiographic studies}

Leads I, II, III, aVR, aVL and aVF were recorded in the right lateral recumbency using an ECG-922 electrocardiograph (Nihon Koden, Tokyo, Japan) [27]. All ECGs were obtained at a paper speed of $50 \mathrm{~mm} / \mathrm{sec}$ and calibration of $10 \mathrm{~mm} / \mathrm{mV}$. First, the electrocardiography were performed in two CXMD (III-302MA, III-303MA) and one normal littermate (III-301MN) dogs at 2, 3, 4, 6, 9, 12, 15, 18 , and 21 months of age, and the heart rate (HR), intervals of $\mathrm{PQ}$ and $\mathrm{QRS}$, and $\mathrm{Q} / \mathrm{R}$ ratios were measured. However, in normal control and in $\mathrm{CXMD}_{\mathrm{J}}, \mathrm{Q}$ waves were not prominent in leads aVR and aVL, therefore, we measured the $\mathrm{Q} / \mathrm{R}$ ratios in leads I, II, III and aVF. Next, we compared the HR, intervals of $\mathrm{PQ}$ and QRS, or Q/R ratios in I, II, III and aVF in eight CXMD J and four normal dogs at 67 months of age.

\section{Echocardiographic studies}

M-mode and two-dimensional echocardiography was performed using an EUB-8000 echocardiograph (Hitachi Medical Corporation, Tokyo, Japan). The thickness of the interventricular septum (IVS) and left ventricular posterior wall (LVPW) at end-diastole, left ventricular internal dimension at end-diastole (LVIDd) and systole (LVIDs), and fractional shortening (FS) were examined on normal and $\mathrm{CXMD}_{\mathrm{J}}$ dogs using M-mode echocardiography. We calculated the M-mode parameters based on multiple measurements of 5 consecutive heart cycles, or 3 or 5 representative heart cycles. We examined the parameters mentioned above and myocardial echogenicity in two $\mathrm{CXMD}_{\text {I }}$ (III-302MA, III-303MA) and one normal littermate (III-301MN) dogs at 2, 3, 4, 6, 9, 12, 15, 18, and 21 months of age. We also examined the parameters mentioned above and myocardial echogenicity in six $\mathrm{CXMD}_{\text {J }}$ (III-D53MA， III-D55MA， III-1803MA， III-D38MA， IIID02MA, III-D08MA) and three normal dogs (III-D56MN, III-1804MN, III-D03MN) at the time point just before euthanasia. Among those dogs, myocardial echogenicity in one CXMD $($ III-D02MA) and its normal littermate (IIID03MN), and another $\mathrm{CXMD}_{\text {J }}$ (III-D08MA) dogs were also examined at various time points.

\section{Macroscopic and histopathological analyses}

All dogs in this study underwent cardiac histological analysis. After a dog was given an overdose of intravenous pentobarbital, the whole heart was removed and immediately fixed in 15\% buffered formalin. Formalin-fixed hearts were cross-sectioned, and samples were taken from the right and left ventricles at the apical papillary muscle and basal levels (each level containing the interventricular septum, anterior wall, lateral wall, and posterior wall). The tissue blocks were embedded in paraffin, and $10 \mu \mathrm{m}$ sections were stained with hematoxylin and eosin or Masson's trichrome stain. Photographs were taken with a DAS Mikroskop LEITZ DMRB microscope (Leica, Wetzlar, Germany), using a digital still camera system HC-2500 (FujiFilm, Tokyo, Japan).

\section{Statistics}

Data are expressed as means +/- SE. Student's $t$ test was used to evaluate differences between the two groups. A $p$ value of less than 0.05 was considered to indicate statistical significance.

\section{Results \\ Clinical profiles of CXMD,}

We recently reported the detailed clinical and histopathological characteristics of CXMD $\mathrm{D}_{\mathrm{J}}$ except for cardiac phenotypes [25]. None of the dogs in the present study showed clinical signs of heart failure, and no murmur was present on auscultation in any $\mathrm{CXMD}_{\mathrm{I}}$ dog examined. We evaluated body and heart weight, the ratio of heart to body weight, and serum $\mathrm{CK}$ in eight $\mathrm{CXMD}_{\mathrm{J}}$ and four normal male dogs aged 6-21 months just before euthanasia (Table 1). There were no differences in body and heart weight and heart/body weight ratio between normal and $\mathrm{CXMD}_{\mathrm{J}}$ dogs. Serum CK levels in the $\mathrm{CXMD}_{\mathrm{J}}$ dogs ranged from 12,500 to $138,000 \mathrm{IU} / 1$. These values were signifi- 
cantly different from those in normal control dogs (60 to 515 IU/1). One 9-month-old CXMD J dog, III-D55MA, did not show any signs of respiratory or cardiac failure. When we tried to record a routine ECG of the dog, the dog struggled to escape from recording and then ceased moving. Immediately afterwards, we recorded ECG and the monitor showed an idioventricular rhythm. The dog died despite attempted cardiopulmonary resuscitation.

\section{Electrocardiographic findings}

The HR and PQ intervals of the affected littermates were no different from those recorded from a normal littermate at 12 months of age, but we detected an increase in HR and a decrease in PQ interval in the affected dogs after 15 months of age (Fig. 1A). The HR and PQ intervals were negatively correlated both in normal and affected dogs (data not shown). The QRS interval in the affected dogs did not differ from that in the normal littermate at any age (Fig. 1A). Prominent deep Q-waves were observed in limb leads II, III, and aVF in some CXMD dogs, but not in the normal littermates, as shown in Fig. $1 \mathrm{~B}$. The Q/R ratios were definitely increased in the affected littermates at 6 months of age or older compared with the normal littermate (Fig. 1C). In all normal and $\mathrm{CXMD}_{\text {J }}$ dogs at 6-7 months of age, the HR, and intervals of PQ and QRS were not different between the two groups of dogs (Fig. 2A), but the $\mathrm{Q} / \mathrm{R}$ ratios in leads II, III, and aVF in the affected dogs were significantly higher than those in the normal dogs (Fig. 2B).

\section{Echocardiographic findings}

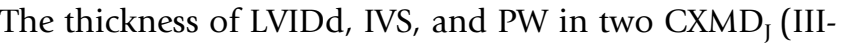
302MA, III-303MA) were not different from those in a normal littermate (III-301MN) by sequential analysis with advancing age (Fig. 3A). Those parameters were not different between other six $\mathrm{CXMD}_{\mathrm{J}}$ and three normal dogs, when examined just before euthanasia (Table 2). FS in III$302 \mathrm{MA}$ decreased with advancing age, and the value $(27.3 \%)$ at 21 months of age was lower than that of the normal littermate, but was within normal range reported previously $[28,29]$. FS in the other seven $\mathrm{CXMD}_{\mathrm{I}}$ were normal, even just before euthanasia (Table 2). A mild hypokinesis of the left ventricular wall was detected in III$302 \mathrm{MA}$ at 21 months of age (Fig. 3B), but any clinical signs had not been developed in the dog.

The sequential studies of myocardial echogenicity with advancing age in III-302MA and in III-303MA demonstrated that the hyperechoic lesions in the left ventricular posterior wall were seen at 12 months of age or older (Fig. $4 \mathrm{~A}$, Table 3 ). In the subsequent examinations of six $\mathrm{CXMD}_{\mathrm{I}}$, we found the hyperechoic lesion in a CXMD $\mathrm{I}_{\mathrm{I}}$ IIID08MA, at 5 months of age (Fig. 4B, Table 3), however the hyperechoic lesion was not detected in other four $\mathrm{CXMD}_{\mathrm{J}}$ at 5 to 7 months old (Fig. 4B, Table 3). One $\mathrm{CXMD}_{\mathrm{I}}$, III-D38MA, did not reveal any hyperechoic lesions when examined at 12 months of age, but has not been examined at 5 to 7 months of age (Table 3). Taken these echocardiographic data, it is considered that the cardiac functions in $C X M D_{\text {J }}$ were basically maintained well by 21 months of age, despite showing hyperechoic lesions of the left ventricle in limited numbers of $\mathrm{CXMD}_{\mathrm{J}}$.

\section{Macroscopic and histopathological findings}

The right and left ventricular walls were examined macroscopically and histopathologically in four normal and eight affected male dogs at the ages shown in Table 1 . The base view of the formalin-fixed heart did not show any macroscopic lesions in III-1803MA at 7 months and III$302 \mathrm{MA}$ at 21 months of age (Fig. 5A) like other affected dogs (data not shown). No histopathological abnormality was found in the posterior wall of the left ventricle of

Table I: Clinical profiles of normal and CXMD, male dogs

\begin{tabular}{|c|c|c|c|c|c|}
\hline & Age (mo) & $\mathrm{BW}(\mathrm{g})$ & $\mathrm{HW}(\mathrm{g})$ & HW/BW (\%) & Serum CK (IU/I) \\
\hline \multicolumn{6}{|l|}{ Normal dogs } \\
\hline III-D56MN & 6 & 12.0 & 95.1 & 0.97 & 515 \\
\hline III-I804MN & 7 & 13.6 & 110.0 & 0.81 & 215 \\
\hline III-D03MN & 14 & 13.1 & 127.0 & 0.84 & 215 \\
\hline III -30I MN & 21 & 14.4 & 120.0 & 0.96 & 60 \\
\hline \multicolumn{6}{|l|}{ CXMD dogs } \\
\hline III-D53MA & 6 & 9.6 & 91.9 & 0.90 & 63,100 \\
\hline III-D55MA & 7 & 10.0 & 80.0 & 0.80 & 42,000 \\
\hline III-I803MA & 9 & 14.4 & 128.6 & 0.69 & 69,100 \\
\hline III-D38MA & 12 & 11.4 & 78.8 & 1.01 & 138,000 \\
\hline III-D02MA & 15 & 9.1 & 92.0 & 0.87 & 17,600 \\
\hline III-D08MA & 15 & 12.0 & 104.3 & 0.97 & 40,700 \\
\hline III-302MA & 21 & 12.4 & 120.0 & 0.86 & 12,500 \\
\hline III-303MA & 21 & 13.9 & 120.0 & 0.80 & 23,000 \\
\hline
\end{tabular}

BW, body weight; HW, heart weight; HW/BW, heart weight/body weight ratio; * $p<0.0$ I normal dogs vs CXMD, dogs 
A
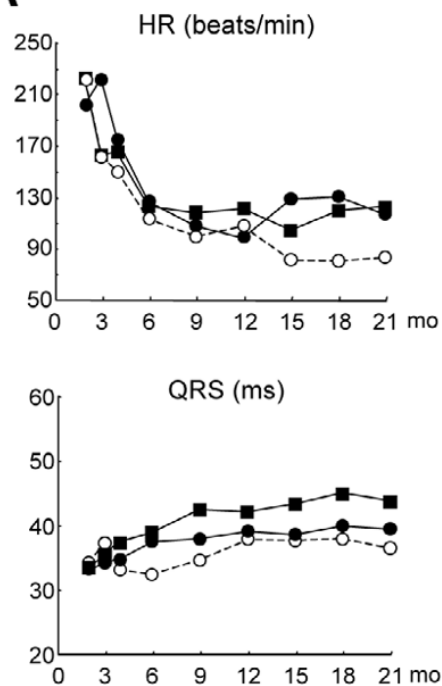

C
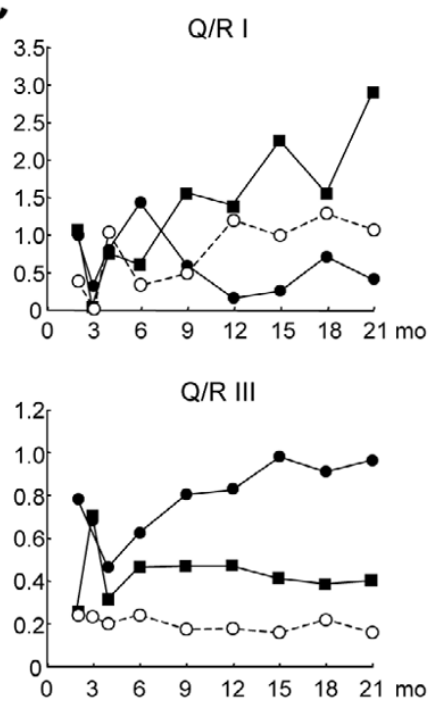
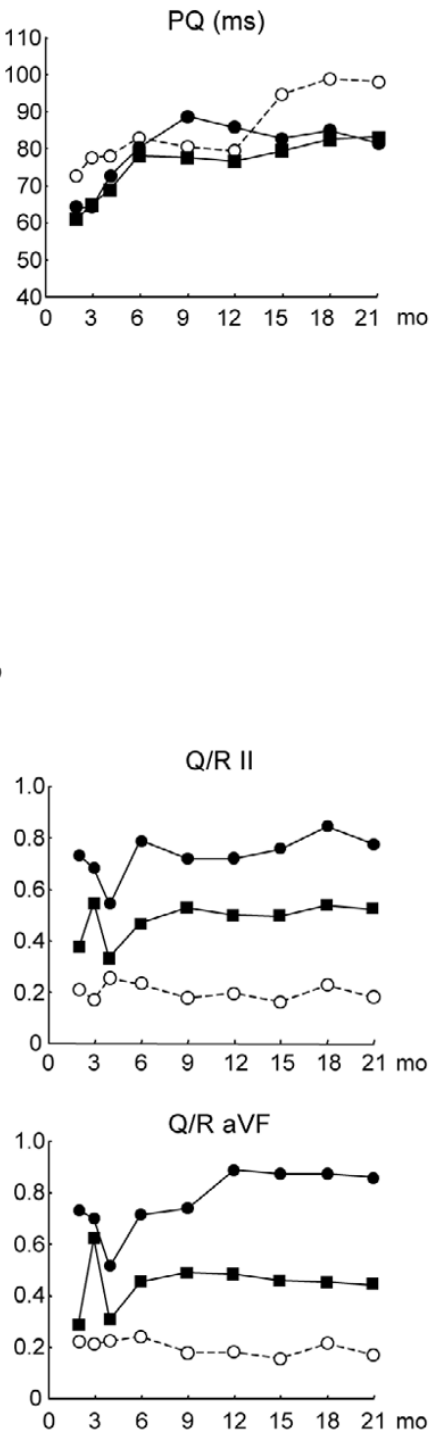
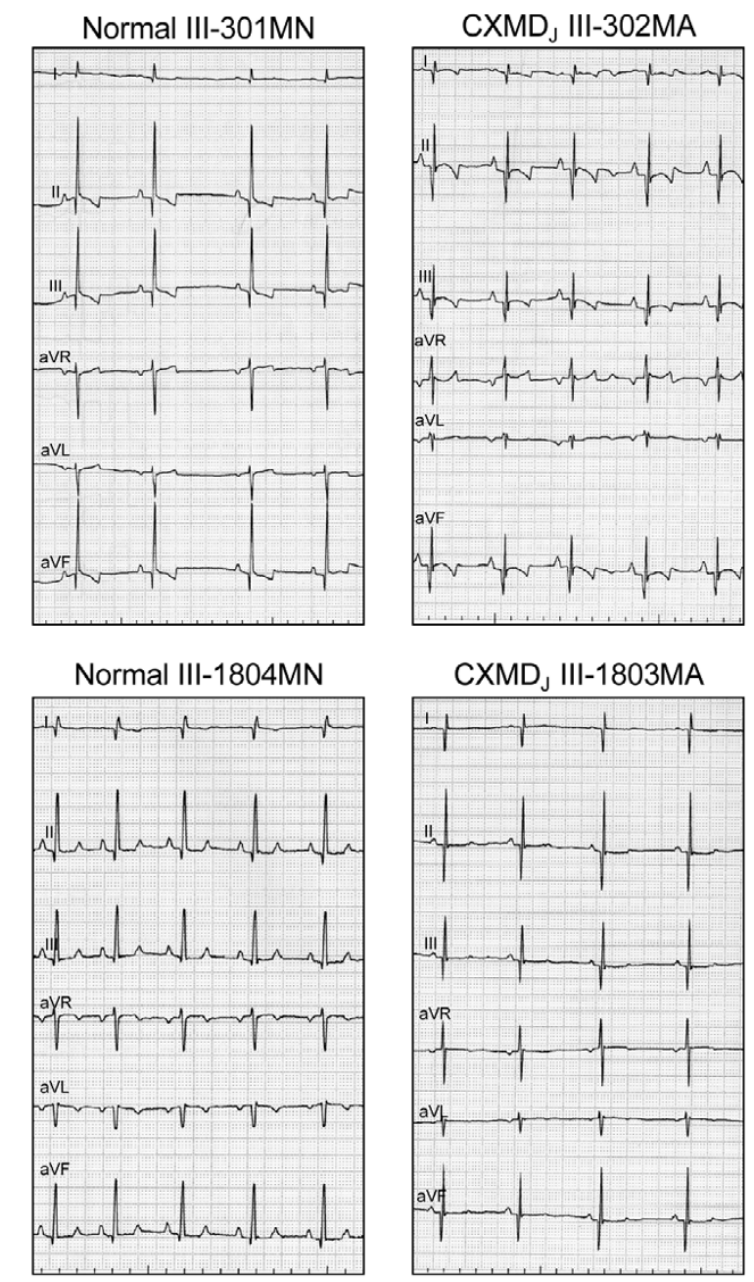

B

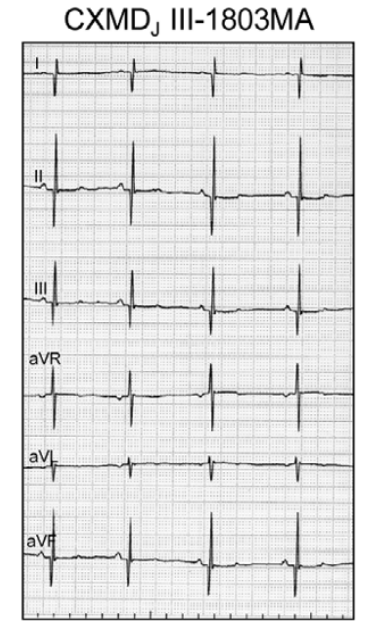

\section{Figure I}

Electrocardiographic findings in CXMD, A: Sequential studies in electrocardiographic parameters with advancing age in normal and CXMD dogs. Heart rate (HR) (beats/min), PQ interval (ms), and duration (ms) of QRS complex on ECG in a normal littermate III-30I MN (open circle), and CXMD dogs III-302MA (closed circle) and III-303MA (closed square) at 2, 3, 4, 6, 9, 12, I5, 18, and 21 months of age. B: Representative ECGs in normal and CXMD, male dogs. ECGs were recorded from normal dogs, III-30IMN and III-I804MN, and CXMD, dogs, III-302MA and III-I803MA, at 6 months of age. Distinct deep Q waves were present in the CXMD, dogs. Leads were recorded at $50 \mathrm{~mm} / \mathrm{s}, 1 \mathrm{~cm} / \mathrm{mV}$. C: Sequential studies in $Q / R$ ratios with advancing age in limb leads I, II, III, and aVF in normal and CXMD dogs. Q/R ratios in limb leads I, II, III, and aVF in a normal littermate III$30 I M N$ (open circle), and the CXMD, dogs III-302MA (closed circle) and III-303MA (closed square) at 2, 3, 4, 6, 9, I2, I5, I8, and 21 months of age.

affected dogs III-1803MA, III-D55MA, and III-D02MA (Fig. 5B), and other affected dogs under the age of 12 months (III-D53MA, III-D38MA). On the other hand, moderate fibrosis in the left ventricular wall, especially on the posterior side, was detected in an affected dog, III302MA, at 21 months (Fig. 5B) as well as in III-D08MA at 

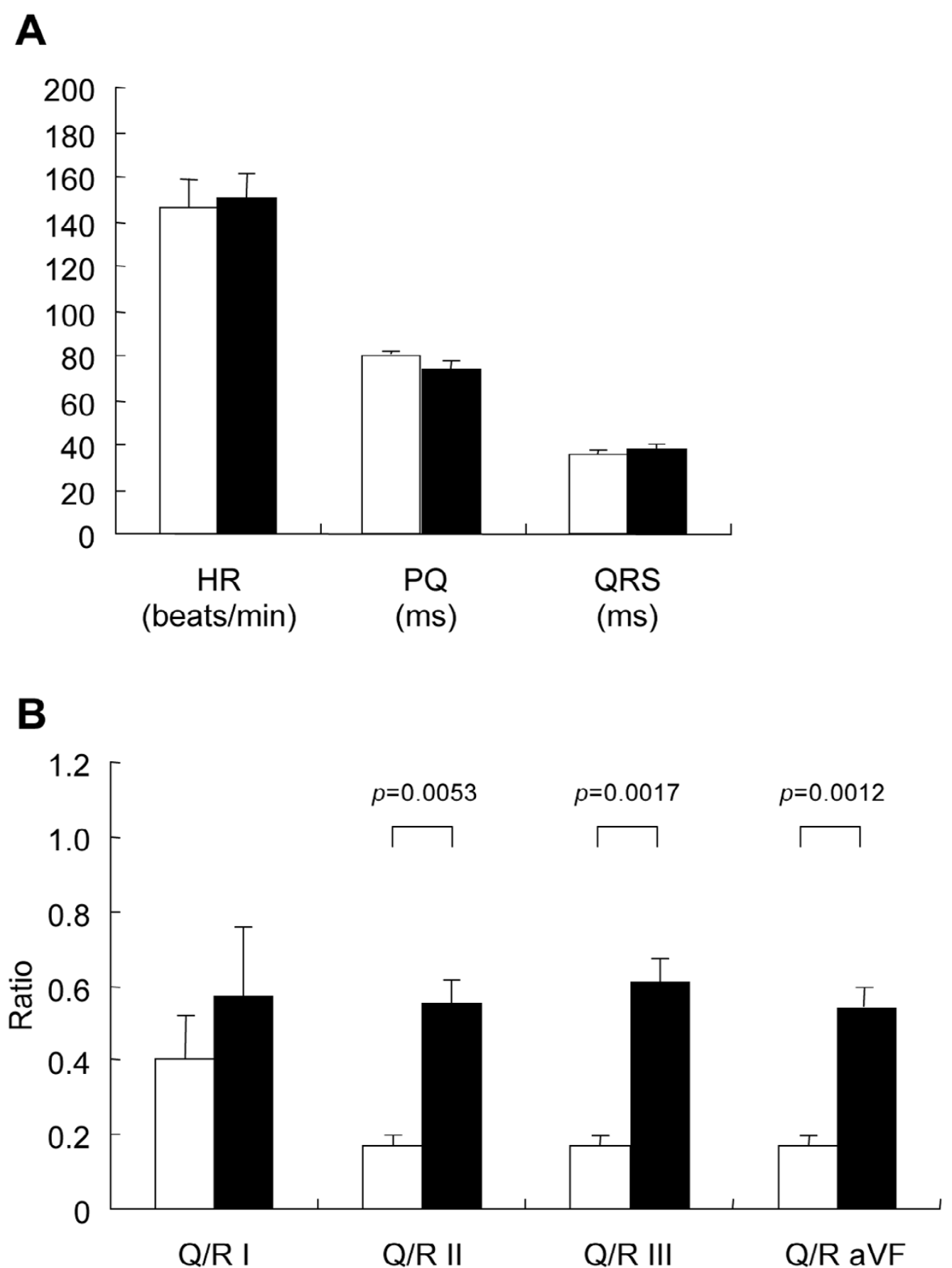

Figure 2

Comparison of electrocardiographic parameters and $Q / R$ ratios between normal and $C X M D_{\text {, dogs at 6-7 }}$ months of age A. Heart rate (HR) (beats/min), PQ interval $(\mathrm{ms})$, and duration of $Q R S$ complex $(\mathrm{ms})$ on ECG in normal $(\mathrm{n}=$ 4) and $\mathrm{CXMD}_{\mathrm{J}}(\mathrm{n}=8)$ dogs at 6-7 months of age. White columns indicate normal dogs, and black columns represent $C X M D_{\text {, }}$ dogs. Bar shows mean +/- SE. B. Q/R ratios in limb leads I (Q/R I), II (Q/R II), III (Q/R III), and aVF (Q/R aVF) on ECG in normal $(n=4)$ and $C X M D_{1}(n=8)$ dogs at 6-7 months of age. White columns indicate normal dogs, and black columns represent CXMD, dogs. Bar shows mean $+/-\mathrm{SE}$. 
A
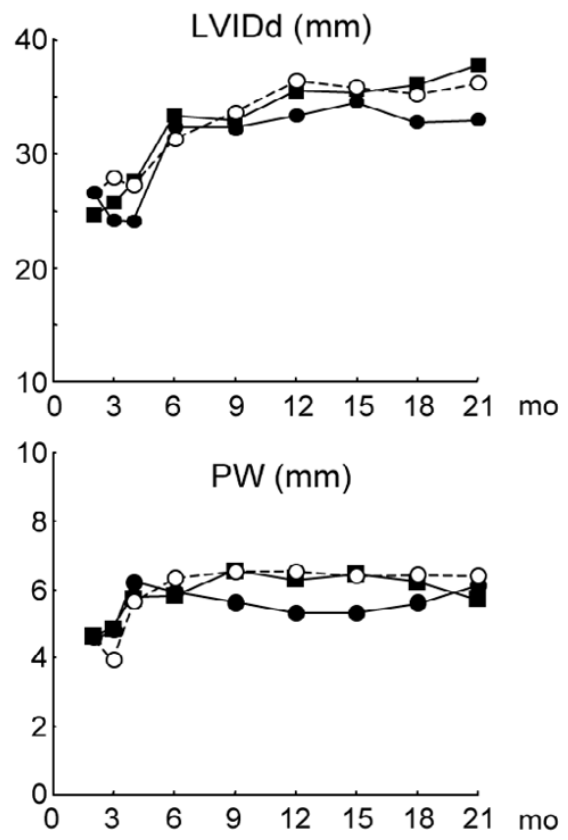

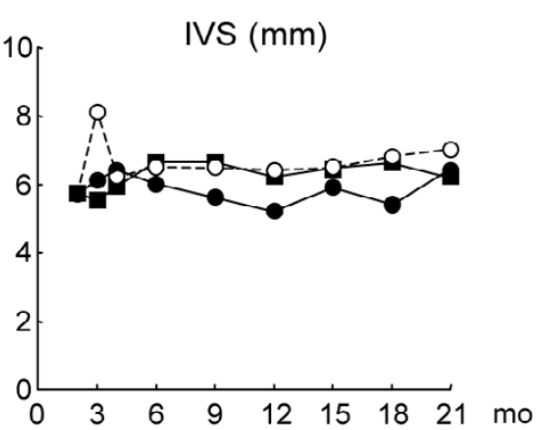

FS (\%)

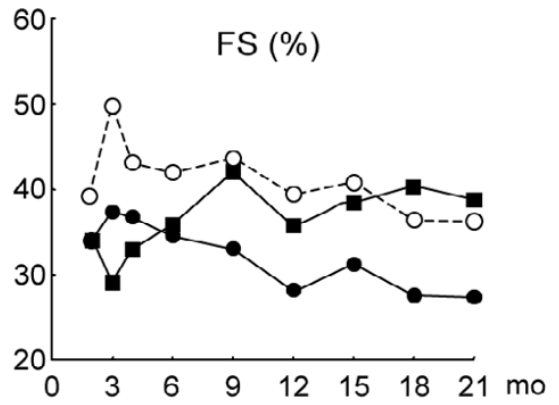

B

Normal
$($ III-301MN, $21 \mathrm{mo})$

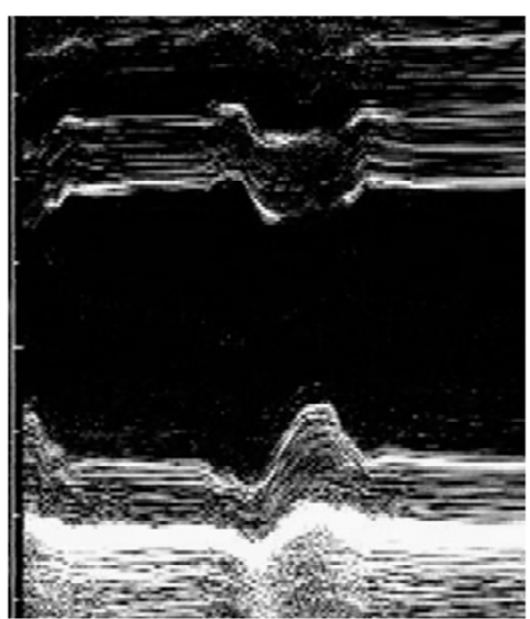

$\mathrm{CXMD}_{J}$ (III-302MA, $21 \mathrm{mo}$ )

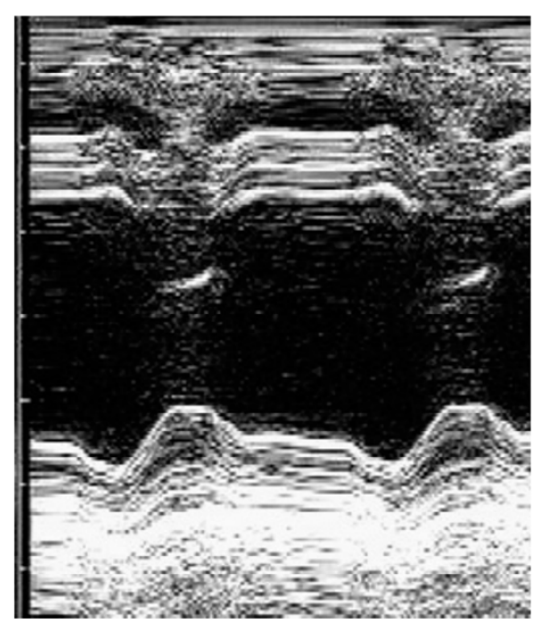

\section{Figure 3}

Cardiac function by echocardiography in CXMD, A: Sequential studies in echocardiographic parameters with advancing age in normal and CXMD dogs. LVIDd (mm), IVS and PW thickness (mm), and FS (\%) in a normal littermate III-30IMN (open circle), and the CXMD dogs III-302MA (closed circle) and III-303MA (closed square) at 2, 3, 4, 6, 9, I2, I5, I8, and 2I months of age. B: M-mode echocardiogram in a normal littermate III-30IMN, compared to the CXMD, dog III-302MA at 21 months of age. Hypokinesis of the left ventricular posterior wall was observed in the CXMD, dog. 
Table 2: Echocardiographic findings in normal and CXMD, male dogs

\begin{tabular}{|c|c|c|c|c|c|c|}
\hline & Age (mo) & LVIDd (mm) & LVIDs (mm) & IVS (mm) & $\mathrm{PW}(\mathrm{mm})$ & FS (\%) \\
\hline \multicolumn{7}{|c|}{ Normal male dogs } \\
\hline III-D56MN & 6 & 34.2 & 12.5 & 6.6 & 5.6 & 63.5 \\
\hline III-I804MN & 7 & 30.7 & 18.9 & 8.2 & 7.4 & 38.4 \\
\hline III-D03MN & 14 & 32.8 & 16.8 & 10.0 & 9.4 & 48.7 \\
\hline III-30I MN & 21 & 36.1 & 23.0 & 7.0 & 6.4 & 36.2 \\
\hline \multicolumn{7}{|c|}{ CXMD, male dogs } \\
\hline III-D53MA & 6 & 29.7 & 19.7 & 8.0 & 7.2 & 33.8 \\
\hline III-D55MA & 7 & 28.7 & 15.4 & 6.3 & 6.3 & 46.5 \\
\hline III-I803MA & 7 & 32.5 & 16.7 & 5.8 & 7.5 & 48.5 \\
\hline III-D38MA & 12 & 30.3 & 18.8 & 8.4 & 5.8 & 37.8 \\
\hline III-D02MA & 15 & 27.5 & 17.3 & 9.1 & 8.8 & 37.0 \\
\hline III-D08MA & 15 & 39.5 & 24.9 & 5.9 & 5.9 & 36.9 \\
\hline III-302MA & 21 & 32.9 & 23.9 & 6.4 & 6.1 & 27.3 \\
\hline III-303MA & 21 & 37.6 & 23.1 & 6.2 & 5.7 & 38.6 \\
\hline
\end{tabular}

Age, age at examination; LVIDd, LV internal dimension diastolic; LVIDs, LV internal dimension systolic; IVS, intraventricular septum thickness; PW, posterior wall thickness; FS, fractional shortening

15 months and III-303MA at 21 months of age (data not shown). We found that the right ventricular walls were kept intact in all $\mathrm{CXMD}_{\mathrm{J}}$ dogs examined.

\section{Discussion}

In electrocardiographic findings, an increased HR and a shortened PQ interval have been reported in both DMD patients [13] and GRMD [22]. These findings were also observed in $\mathrm{CXMD}_{\mathrm{J}}$ dogs. Increased sympathetic activity and decreased parasympathetic activity have been observed in DMD patients and are associated with disease progression [30]; therefore, autonomic dysfunction in dystrophin deficiency might affect these parameters. It has been reported that HR is negatively correlated with PQ interval in normal Beagle dogs and it may be ascribed to a parasympathetic input at the level of the AV node [31]. The negative correlation between $\mathrm{HR}$ and PQ intervals was also found in affected dogs, indicating the parasympathetic input was maintained well even in affected dogs at AV node level. The QRS duration was within normal limits in the CXMD dogs, which is compatible with most cases of DMD [13]. Another peculiar electrocardiographic finding in $\mathrm{DMD}$ is the deep and narrow $\mathrm{Q}$-waves in $\mathrm{I}, \mathrm{aVL}$ and V6 or in II, III and aVF $[10,13,16,32]$. CXMD ${ }_{\text {J }}$ dogs also showed prominent $\mathrm{Q}$-waves and increases in the $\mathrm{Q} / \mathrm{R}$ ratio in leads II, III, and aVF, findings that are consistent with those in GRMD [23]. In all CXMD ${ }_{\text {I }}$ dogs examined, the distinct deep Q-waves were recognized by 6-7 months of age, which is earlier than the other abnormal electrocardiographic parameters, and the $\mathrm{Q} / \mathrm{R}$ ratio in affected dogs remained high from 6 to 21 months of age. Actually, the prominent $\mathrm{Q}$-wave and increase in $\mathrm{Q} / \mathrm{R}$ ratio were also detected in some of the CXMD Iogs at around 2 months of age (Fig. 1C), but it is difficult to evaluate the degree of the $Q / R$ ratio increase before 3 months of age because the
QRS vector is almost exclusively directed to the right and varies significantly in the weeks after birth [33]. A previous report described GRMD dogs ranging from 6 months to $>2$ years as having deep Q-waves and increased Q/R ratios in leads II, III, and aVF [23]. The Q-waves, however, might have been seen earlier and regarded as normal variants or not have been considered important for the reasons mentioned above.

Hyperechoic lesions indicating myocardial fibrosis in the posterobasal left ventricular wall have been detected by echocardiography in GRMD dogs as well as DMD patients $[22,23]$. Moise et al. reported that hyperechoic lesions were first detected in eight of eleven GRMD dogs (73\%) by $6-7$ months of age and that they correlated with histologically recognizable areas of mineralization and corresponded to the progression of fibrosis [23]. In our study, one of eight of $C X M D_{\text {J }}$ dogs showed a hyperechoic lesion in the left ventricular posterior wall, but the rest had not by the age of 6-7 months (Table 3, Fig. 4). The hyperechoic lesion in the left ventricular posterior walls was detected in both III-302MA and III-303MA, but not early as 12 months of age (Table 3 ). The results of echocardiography indicated that the cardiac involvement in $\mathrm{CXMD}_{\mathrm{J}}$ is milder than that in GRMD. Echocardiography did not reveal particular left ventricular dysfunction in any CXMD I dog by 21 months of age, but a mild hypokinesis of the left ventricular wall was observed in III-302MA at 21 months of age (Fig. 3B). The dysfunction found in the dog, however, was mild and the dog had no cardiac symptom. Moise et al. reported that three of the six GRMD dogs $>2$ years of age showed a decrease in fractional shortening, but did not mention at what age the abnormal cardiac findings appeared. 
A

Normal (III-301MN) CXMDJ (III-302MA)

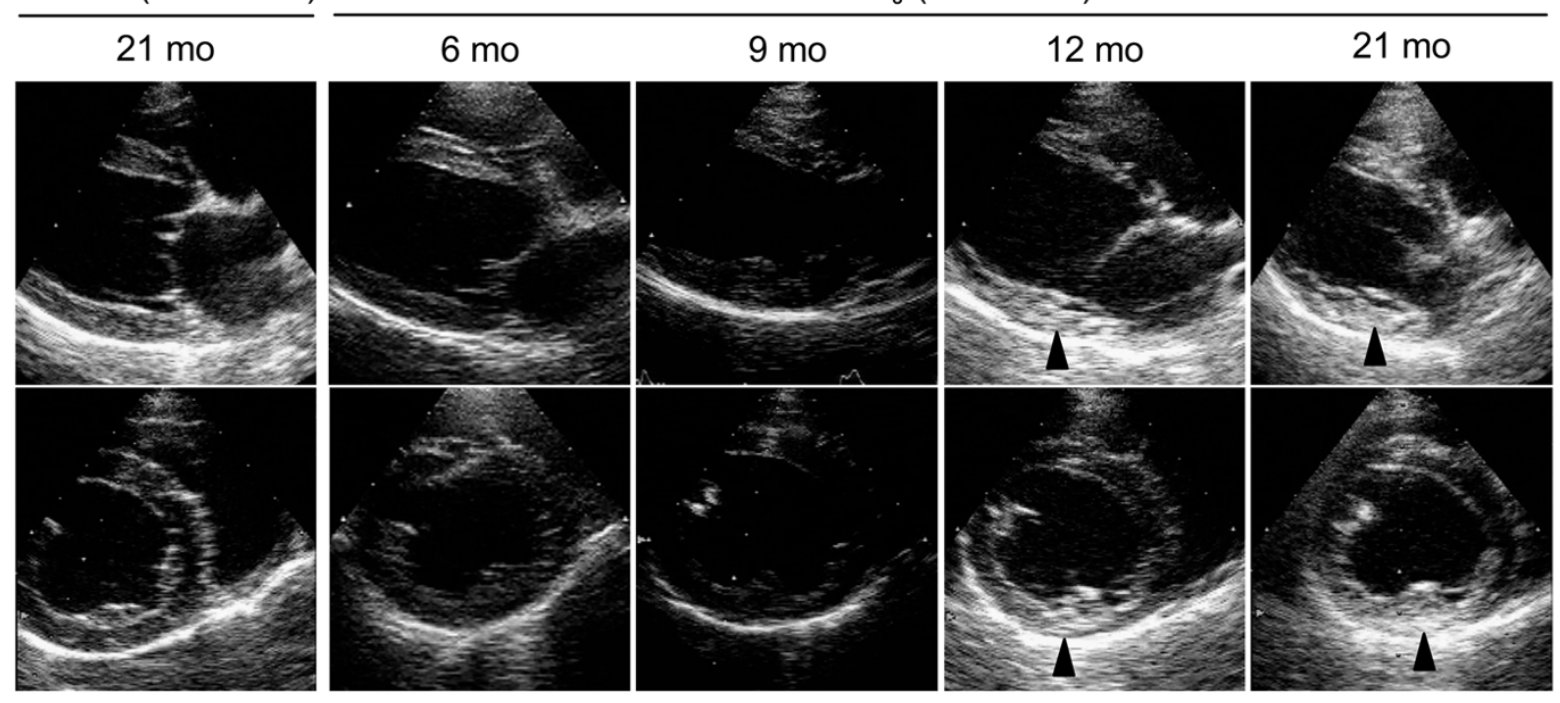

B

III-301MN, 6 mo

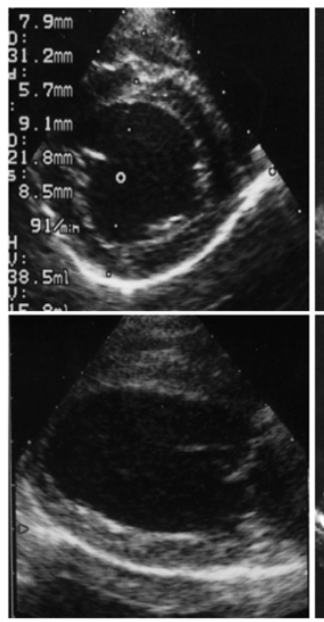

III-D53MA, 6 mo

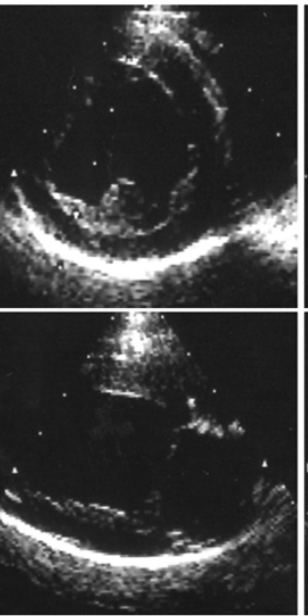

III-D55MA, 7 mo

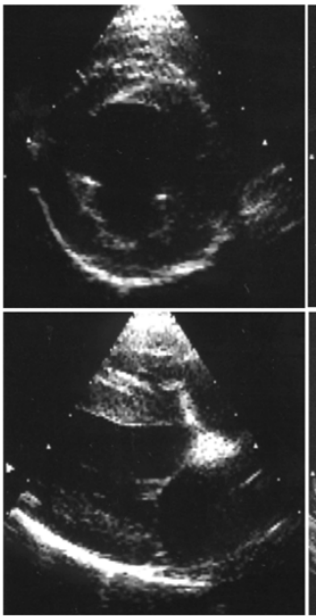

III-1803MA, 7 mo

III-D08MA, 7 mo

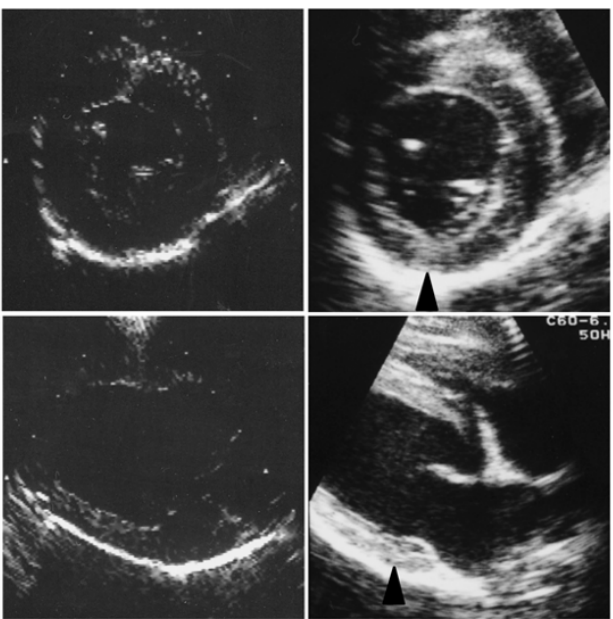

Figure 4

Echogenicity in CXMD, A: Sequential studies in echogenicity with advancing age by two-dimensional echocardiography in a normal dog III-30IMN, and a CXMD dog III-302MA, at 6-2I months of age. Hyperechoic lesions (arrowheads) of the left ventricular posterior wall were detected in the CXMD, dog at 12 months of age and older.B: Two-dimensional echocardiograms of a normal dog III-30IMN at 6 months of age, and four CXMD, dogs III-D53MA, III-D55MA, III-I803MA, and III-D08MA at 6 to 7 months of age. The hyperechoic lesion (arrowhead) was detected only in the left ventricular posterior wall of III-D08MA.

Previous studies of morphology in GRMD showed that myocardial involvement is initially found in the left pos- terobasal ventricular wall, similar to that of patients with DMD [21-23]. Valentine et al. reported that GRMD dogs 
Table 3: Echogenicity of left ventricular posterior wall in normal and CXMD, male dogs

\begin{tabular}{|c|c|c|c|c|c|c|c|c|c|c|c|c|c|c|c|c|c|c|c|c|}
\hline & \multicolumn{20}{|c|}{ Months of age $(\mathrm{mo})$} \\
\hline & 2 & 3 & 4 & 5 & 6 & 7 & 8 & 9 & 10 & 11 & 12 & 13 & 14 & 15 & 16 & 17 & 18 & 19 & 20 & 21 \\
\hline \multicolumn{21}{|l|}{ Normal dogs } \\
\hline III-D56MN & & & & & $(-)^{*}$ & & & & & & & & & & & & & & & \\
\hline III-I804MN & & & & & $(-)$ & $(-)^{*}$ & & & & & & & & & & & & & & \\
\hline III-D03MN & $(-)$ & & & $(-)$ & & $(-)$ & & & $(-)$ & & & $(-)$ & $(-)^{*}$ & & & & & & & \\
\hline III-30I MN & $(-)$ & $(-)$ & $(-)$ & & $(-)$ & & & $(-)$ & & & $(-)$ & & & $(-)$ & & & $(-)$ & & & $(-)^{*}$ \\
\hline \multicolumn{21}{|l|}{$C X M D_{\text {J dogs }}$} \\
\hline III-D53MA & & & & & $(-)^{*}$ & & & & & & & & & & & & & & & \\
\hline III-D55MA & & & & & & $(-)$ & & $*$ & & & & & & & & & & & & \\
\hline III-I803MA & & & & & $(-)$ & $(-)^{*}$ & & & & & & & & & & & & & & \\
\hline III-D38MA & & & & & & & & & & & $(-)^{*}$ & & & & & & & & & \\
\hline III-D02MA & $(-)$ & & & $(-)$ & & $(-)$ & & & & & $(-)$ & $(-)$ & & $(-)^{*}$ & & & & & & \\
\hline III-D08MA & $(-)$ & & & $(+)$ & & $(+)$ & & & & & $(+)$ & $(+)$ & & $(+)^{*}$ & & & & & & \\
\hline III-302MA & $(-)$ & $(-)$ & $(-)$ & & $(-)$ & & & $(-)$ & & & $(+)$ & & & $(+)$ & & & $(+)$ & & & $(+)^{*}$ \\
\hline III-303MA & $(-)$ & $(-)$ & $(-)$ & & $(-)$ & & & $(-)$ & & & $(+)$ & & & $(+)$ & & & $(+)$ & & & $(+)^{*}$ \\
\hline
\end{tabular}

Hyperechoic lesion +, positive; -, negative; Asterisk in each CXMD, dog shows age at euthanasia.

at 6.5 months of age had acute severe lesions with focal myocardial mineralization associated macrophages and giant cells in the left ventricular papillary muscle and left ventricular wall [22]. Moreover, GRMD dogs at 12 months of age or older demonstrated prominent myocardial fibrosis in more widespread lesions [22]. The myocardial fibrosis of the left ventricular wall in the older stage of CXMD dogs was consistent with that in DMD patients and GRMD dogs. The change was detected at 15 months of age or older in the CXMD (III-D08MA, III-302MA, and III-303MA), although III-D08MA showed a hyperechoic lesion at 5 months of age or older (Table 3 ). The cardiac involvement in $\mathrm{CXMD}_{\mathrm{J}}$, therefore, was milder and slowly progressed than that in GRMD, although a longer period evaluation of large numbers of $\mathrm{CXMD}_{\mathrm{J}}$ will be needed to conclude the mild cardiac phenotypes of $\mathrm{CXMD}_{\mathrm{J}}$.

Why is the cardiac involvement in $\mathrm{CXMD}_{\mathrm{J}}$ milder than that in GRMD? Valentine et al. reported that skeletal muscle involvement in small dystrophic dogs was milder than that in large ones [19]. Several reports on dystrophic features have hypothesized that the clinical severity may be associated with growth rate [34] or muscle fiber diameter [35]. Living in a cage rather than running free could also affect the cardiac phenotypes of $\mathrm{CXMD}_{\mathrm{J}}$ because physical exercise promotes cardiac involvement in dystrophindeficient $m d x$ mice [36]. The difference in the genetic background between GRMD, golden retriever and $\mathrm{CXMD}_{\mathrm{J}}$, Beagle might also affect the disease progression.

The prominent deep Q-waves seen in both DMD and GRMD have been attributed to a reduction in or a loss of electromotive force caused by scarring of the posterobasal region of the left ventricle $[8,9,17]$. In our study, the deep $\mathrm{Q}$-waves and increases in the $\mathrm{Q} / \mathrm{R}$ ratio in $\mathrm{CXMD}_{\text {J }}$ preceded the lesions seen on echocardiogram and histopathology, as shown in Fig. 6. Considering this result, the origin of the distinctive Q-waves might not be associated with the myocardial lesion in the posterobasal left ventricular wall. It has recently been reported that expression of a transgene in $m d x$ mice for neuronal nitric oxide synthase (nNOS), which occurs as a secondary loss in dystrophin deficiency, decreased cardiac inflammation and fibrosis resulting in amelioration of both cardiac function and electrocardiographic abnormalities, including deep Q-waves [37]. Perloff et al. suggested that the alteration of a particular ionic current by lack of specific membrane proteins associated with dystrophin might participate in electrocardiographic changes [17]. We will not therefore deny that minimal myocardial damage could be associated with the pathogenesis of deep Q-waves, but our results suggest that an investigation of the conduction and cardiovascular systems will also be needed to explore the pathophysiology of the deep Q-waves in dystrophin-deficient heart. In this regard, $\mathrm{CXMD}_{\mathrm{J}}$ will be very useful to elucidate aspects of the dystrophin-deficient heart, but we may recognize that a longer period of time would be required to complete cardiac phenotypes in $\mathrm{CXMD}_{\mathrm{J}}$.

\section{Conclusion}

We demonstrated that the cardiac phenotypes of $\mathrm{CXMD}_{\mathrm{J}}$ are comparable to but milder than those of GRMD. Furthermore, we found for the first time that the distinct deep $\mathrm{Q}$-waves precede detection of the left ventricular posterobasal lesion by echocardiography or histopathology. $\mathrm{CXMD}_{\mathrm{J}}$ may provide not only new insights into the mech- 
A

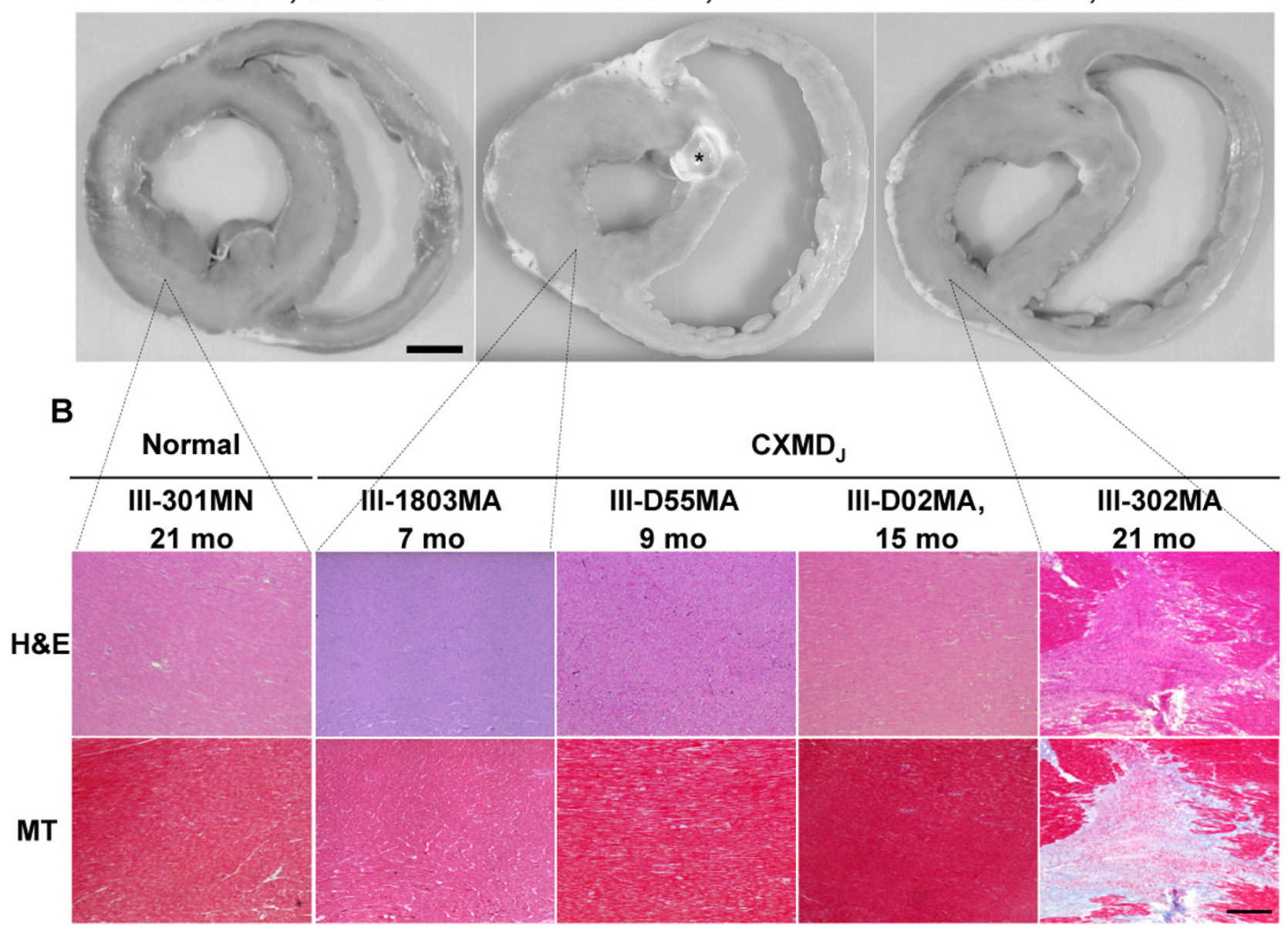

\section{Figure 5}

Macroscopic and histopathological findings in CXMD, hearts A. Macroscopic examinations of the base of the formalinfixed hearts of a normal littermate III-30IMN at 2 I months and CXMD, dogs, III-I803MA at 7 months and III-302MA at 2I months of age. *Aortic valve. Bar shows I cm. B. Hematoxylin and eosin (H\&E) and Masson's trichrome (MT) staining for histopathological evaluation of the left ventricular posterior wall in a normal littermate, III-30IMN at $2 I$ months and the CXMD, dogs, III-I 803MA at 7 months, III-D55MA at 9 months, III-D02MA at I5 months, and III-302MA at $2 \mathrm{I}$ months of age. Posterior walls of left ventricles of both III-D55MA and III-D02MA were macroscopically normal (data not shown). Bar shows $200 \mu \mathrm{m}$.

anisms causing the abnormal Q-waves but also more information on the pathogenesis in the dystrophin-deficient heart.

\section{Competing interests}

The author(s) declare that they have no competing interests.

\section{Authors' contributions}

NY and NU carried out the electrocardiographic, echocardiographic, and pathological examination and drafted the manuscript. YF performed the electrocardiographic study. MY, KY and MRW participated in the necropsy and pathological examination. MN, YS, MT and AT participated in the maintenance of the dog colony and the design of the study. NM performed the pathological examination. YW participated in the design of the study. AN participated in the statistical analysis and drafted manuscript. ST participated in the design, planning and coordination of the study. All authors read and approved the final manuscript.

\section{Acknowledgements}


GRMD

Increase in Q/R ratio in leads II, III and aVF

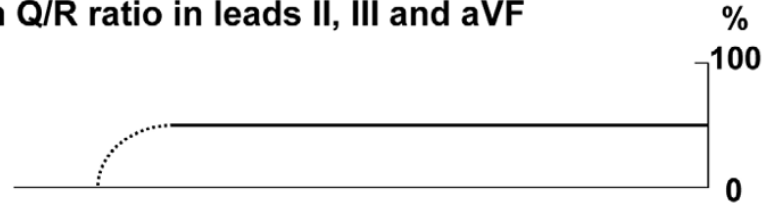

$\%$

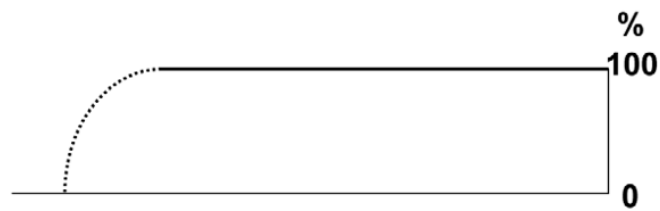

CXMDJ

Hyperechoic lesion in posterior wall by echocardiography
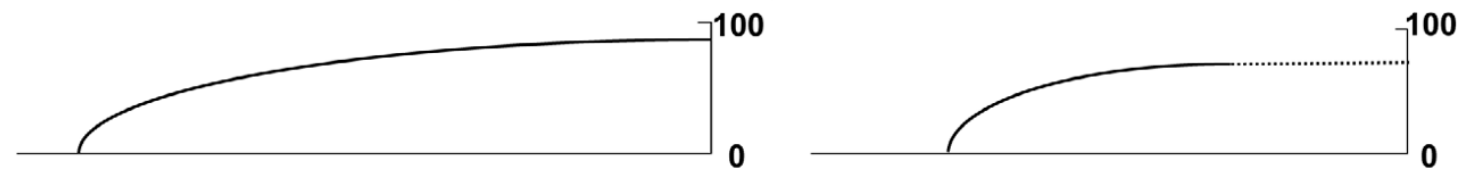

Fibrotic change in posterior wall by pathology

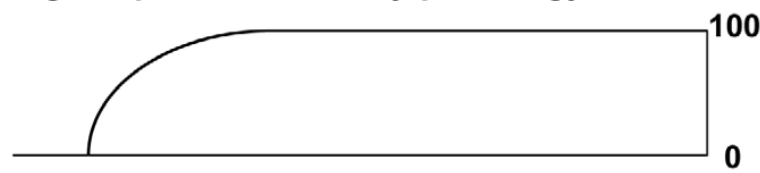

$\begin{array}{lllllllllllll}2 & 4 & 6 & 8 & 10 & 12 & 14 & 16 & 18 & 20 & 22 & 24 & \mathrm{mo}\end{array}$

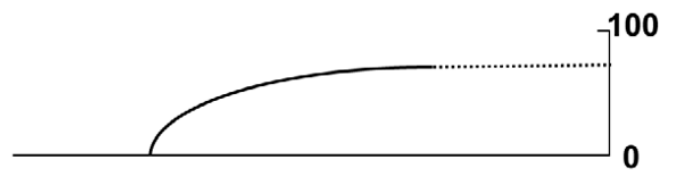

$\begin{array}{lllllllllll}2 & 4 & 6 & 8 & 10 & 12 & 14 & 16 & 18 & 20 & \mathrm{mo}\end{array}$

\section{Figure 6}

Comparison of cardiac involvement between GRMD and CXMD, with advancing age. Subjects were compared as follows: increase in Q/R ratio in leads II, III, and aVF in ECG, hyperechoic lesion in posterior wall by echocardiography, and fibrotic change in left ventricular posterior wall by pathology. The data on GRMD was based on the previous literature [2224]. It is difficult to evaluate $Q / R$ ratio in early stage of GRMD and CXMD. It is also difficult to evaluate hyperechoic lesion in echocardiogram and fibrotic change in pathology at late stage of $C X M D_{\text {, }}$ due to small numbers of examination $(n<3)$.

We thank Hideki Kita, Shin'ichi Ichikawa, Yumiko Yahata, and Kazue Kinoshita (JAC, Inc., Tokyo) for maintaining the dogs, Yoshikuni Tanioka (Central Institute for Experimental Animals, Kawasaki) for his support and valuable suggestions, and Ryoko Nakagawa (Department of Molecular Therapy, National Institute of Neuroscience, NCNP, Tokyo) for her technical assistance. This study was supported by Health Sciences Research Grants for Research on Psychiatric and Neurological Diseases and Mental Health (HI2-kokoro-025, HI5-kokoro-02I, HI8-kokoro-019), the Human Genome and Gene Therapy (HI3-genome-00I, HI6-genome-003) from the Ministry of Health, Labor and Welfare of Japan, and Grants-in-Aid for Scientific Research from the Ministry of Education, Science, Sports and Culture of Japan (to S.T.).

\section{References}

I. Koenig M, Hoffman EP, Bertelson CJ, Monaco AP, Feener C, Kunkel LM: Complete cloning of the Duchenne muscular dystrophy (DMD) cDNA and preliminary genomic organization of the DMD gene in normal and affected individuals. Cell 1987, 50:509-517.

2. Cullen MJ, Mastaglia FL: Morphological changes in dystrophic muscle. Br Med Bull 1980, 36: |45-I52.

3. Ervasti JM, Ohlendieck K, Kahl SD, Gaver MG, Campbell KP: Deficiency of a glycoprotein component of the dystrophin complex in dystrophic muscle. Nature 1990, 345:315-319.
4. Gilroy J, Cahalan JL, Berman R, Newman M: Cardiac and pulmonary complications in Duchenne's progressive muscular dystrophy. Circulation 1963, 27:484-493.

5. Moser H: Duchenne muscular dystrophy: pathogenetic aspects and genetic prevention. Hum Genet 1984, 66: I7-40.

6. Mukoyama M, Kondo K, Hizawa K, Nishitani H: Life spans of Duchenne muscular dystrophy patients in the hospital care program in Japan. J Neurol Sci 1987, 8 I:I55-I58.

7. Eagle M, Baudouin SV, Chandler C, Giddings DR, Bullock R, Bushby $\mathrm{K}$ : Survival in Duchenne muscular dystrophy: improvements in life expectancy since 1967 and the impact of home nocturnal ventilation. Neuromuscul Disord 2002, I 2:926-929.

8. Perloff JK, Roberts WC, de Leon AC Jr, O'Doherty D: The distinct electrocardiogram of Duchenne's progressive muscular dystrophy. An electrocardiographic-pathologic correlative study. Am J Med 1967, 42: I79-188.

9. Sanyal SK, Johnson WW, Thapar MK, Pitner SE: An ultrastructural basis for electrocardiographic alterations associated with Duchenne's progressive muscular dystrophy. Circulation 1978 , 57: I I22-I I 29.

10. Perloff JK, de Leon AC Jr, O'Doherty D: The cardiomyopathy of progressive muscular dystrophy. Circulation 1966, 33:625-648.

II. Frankel KA, Rosser RJ: The pathology of the heart in progressive muscular dystrophy: epimyocardial fibrosis. Hum Pathol 1976, 7:375-386.

12. James TN: Observation on the cardiovascular involvement, including the cardiac conduction system, in progressive muscular dystrophy. Am Heart J 1962, 63:48-56. 
13. Finsterer J, Stöllberger C: The heart in human dystrophinopathies. Cardiology 2003, 99:1-19.

14. Farah MG, Evans EB, Vignos PJ jr: Echocardiographic evaluation of left ventricular function in Duchenne's muscular dystrophy. Am J Med 1980, 69:248-254.

15. D'Orsogna L, O'Shea JP, Miller G: Cardiomyopathy of Duchenne muscular dystrophy. Pediatr Cardiol 1988, 9:205-2/3.

16. Perloff JK: Cardiac rhythm and conduction in Duchenne's muscular dystrophy: a prospective study of 20 patients. J Am Coll Cardiol 1984, 3:1263-1268.

17. Perloff JK, Moise NS, Stevenson WG, Gilmour RF: Cardiac electrophysiology in Duchenne muscular dystrophy: From basic science to clinical expression. J Cardiovasc Electrophysiol 1992, 3:394-409.

18. Goodwin FC, Muntoni F: Cardiac involvement in muscular dystrophies: molecular mechanisms. Muscle Nerve 2005, 32:577-588

19. Cooper BJ, Winand NJ, Stedman H, Valentine BA, Hoffman EP, Kunkel LM, Scott MO, Fischbeck KH, Kornegay JN, Avery RJ, Williams JR, Schmickel RD, Sylvester JE: The homologue of the Duchenne locus is defective in $\mathbf{X}$-linked muscular dystrophy of dogs. Nature 1988, 334:154-156.

20. Valentine BA, Cooper BJ, de Lahunta A, O'Quinn R, Blue JT: Canine $X$-linked muscular dystrophy. An animal model of Duchenne muscular dystrophy: clinical studies. J Neurol Sci 1988, 88:69-8I

21. Valentine BA, Winand NJ, Pradhan D, Moise NS, de Lahunta A, Kornegay JN, Cooper BJ: Canine X-linked muscular dystrophy as an animal model of Duchenne muscular dystrophy: a review. Am J Med Genet I 992, 42:352-356.

22. Valentine BA, Cummings JF, Cooper BJ: Development of Duchenne-type cardiomyopathy. Morphologic studies in a canine model. Am J Pathol 1989, I35:67I-678.

23. Moise NS, Valentine BA, Brown CA, Erb HN, Beck KA, Cooper BJ, Gilmour RF: Duchenne's cardiomyopathy in a canine model: electrocardiographic and echocardiographic studies. J Am Coll Cardiol 1991, 17:812-820.

24. Shimatsu $Y$, Katagiri K, Furuta T, Nakura M, Tanioka Y, Yuasa K Tomohiro M, Kornegay JN, Nonaka I, Takeda S: Canine X-linked muscular dystrophy in Japan (CXMD). Exp Anim 2003, 52:93-97.

25. Shimatsu Y, Yoshimura M, Yuasa K, Urasawa N, Tomohiro M, Nakura M, Tanigawa M, Nakamura A, Takeda S: Major clinical and histopathological characteristics of canine $\mathbf{X}$-linked muscular dystrophy in Japan, CXMD. Acta Myol 2005, 24:145-154.

26. Honeyman K, Carville KS, Howell JM, Fletcher S, Wilton SD: Development of a snapback method of single-strand conformation polymorphism analysis for genotyping Golden Retrievers for the X-linked muscular dystrophy allele. Am J Vet Res 1999, 60:734-737.

27. Tilley LP: Basic canine and feline electrocardiography. Can Vet J 1981, 22:23-25.

28. Crippa L, Ferro E, Melloni E, Brambilla P, Cavalletti E: Echocardiographic parameters and indices in the normal Beagle dog. Lab Anim 1992, 26:190-195.

29. Cornell CC, Kittleson MD, Della Torre P, Häggström J, Lombard CW, Pedersen HD, Vollmar A, Wey A: Allometric scaling of $\mathbf{M}$ mode cardiac measurements in normal adult dogs. J Vet Intern Med 2004, I 8:3II-32I.

30. Yotsukura M, Fujii K, Katayama A, Tomono Y, Ando H, Sakata K, Ishihara $T$, Ishikawa $K$ : Nine-year follow-up study of heart rate variability in patients with Duchenne-type progressive muscular dystrophy. Am Heart J 1998, 136:289-296.

31. Hanton G, Rabemampianina Y: The electrocardiogram of the beagel dog: reference values and effect of sex, genetic strain, body position and heart rate. Lab Anim 2006, 40:123-136.

32. Sanyal SK, Johnson WW: Cardiac conduction abnormalities in children with Duchenne's progressive muscular dystrophy: electrocardiographic features and morphologic correlates. Circulation 1982, 66:853-863.

33. Trautvetter E, Detweiler DK, Patterson DF: Evolution of the electrocardiogram in young dogs during the first 12 weeks of life. J Electrocardiol | 981, 14:267-273.

34. Zats M, Betti RT: Benign Duchenne muscular dystrophy in a patient with growth hormone deficiency. Am J Med Genet 1986, 24:567-572.
35. Braund KG, McGuire JA, Lincoln CE: Observations on normal skeletal muscle of mature dogs: a cytochemical, histochemical, and morphometric study. Vet Pathol 1982, 19:577-595.

36. Nakamura A, Yoshida K, Takeda S, Dohi N, lkeda S: Progression of dystrophic features and activation of mitogen-activated protein kinases and calcineurin by physical exercise, in hearts of mdx mice. FEBS Lett 2002, 520:18-24.

37. Wehling-Henricks M, Jordan MC, Roos KP, Deng B, Tidball JG: Cardiomyopathy in dystrophin-deficient hearts is prevented by expression of a neuronal nitric oxide synthase transgene in the myocardium. Hum Mol Genet 2005, I 4:1921-1933.

\section{Pre-publication history}

The pre-publication history for this paper can be accessed here:

http://www.biomedcentral.com/1471-2261/6/47/prepub
Publish with Bio Med Central and every scientist can read your work free of charge

"BioMed Central will be the most significant development for disseminating the results of biomedical research in our lifetime. "

Sir Paul Nurse, Cancer Research UK

Your research papers will be:

- available free of charge to the entire biomedical community

- peer reviewed and published immediately upon acceptance

- cited in PubMed and archived on PubMed Central

- yours - you keep the copyright 